\title{
Supporting Information - Bioaccumulation potential of CPs in aquatic organisms: Uptake and depuration in Daphnia magna
}

Mafalda Castro*, Anna Sobek, Bo Yuan, Magnus Breitholtz.

Department of Environmental Science and Analytical Chemistry (ACES), Stockholm University, 106-91 Stockholm, Sweden

25 pages

11 Tables

7 Figures 


\section{Calculation of modelled $\log$ BAF values}

Modelled log BAF values were obtained using U.S. Environmental Protection Agency's $K_{\text {ow }}$ (based) Aquatic Bioaccumulation Model (KABAM version 1.0 (2009))*, which is based on an aquatic food web bioaccumulation model published by Arnot and Gobas. ${ }^{1}$ The model was adapted for filter feeders.

For the calculation of the modelled BAF value, the $K_{\text {ow }}$ input value $\left(\mathrm{T}=20^{\circ} \mathrm{C}\right)$ was for each mixture was estimated using the US EPA EPISuite software package (version 4.1) predicted by KOWWIN and BCFBAF tools. The $K_{\text {ow }}$ was calculated for 224 congeners (from $\mathrm{C}_{9} \mathrm{Cl}_{3}$ to $\mathrm{C}_{31} \mathrm{Cl}_{12}$ ). For each molecular formula, one isomer was selected with the following assumptions: i) equally distributed chlorine substitution through the carbon chain starting from the middle of the chain and ii) one chlorine per carbon atom. The reconstructed congener composition of each $\mathrm{CP}$ technical mixture was used to calculate the relative contribution $(\mathrm{RC})$ of each congener in the whole mixture, which multiplied by each congener specific $K_{\text {ow }}$, gave a final estimated $K_{\text {ow }}$ (Equation (S1)).

$K_{\text {ow }}($ estimated $)=\sum K_{\text {ow }_{\text {congener } 1}} \times R C_{\text {congener } 1}+\ldots+K_{\text {ow }_{\text {congener } n}} \times R C_{\text {congener } n}$

Feeding rate was measured through a simple 24 hour feeding test, instead of modelled through KABAM. The feeding test performed in this work was done according to Barata et al. (2008), where feeding rate was measured with an initial food concentration of $4 \mu \mathrm{g} \mathrm{C} \mathrm{mL}{ }^{-1}$. Feeding rate of the animals (model input value) was $7.7 \times 10^{-7} \mathrm{~kg}$ algae day ${ }^{-1}$.

"https://www.epa.gov/pesticide-science-and-assessing-pesticide-risks/kabam-version-10-usersguide-and-technical-7\#A2 
Table S1. Average recoveries (\%) obtained for water and daphnia samples for the different CP mixtures.

\begin{tabular}{ccccccc} 
& $\begin{array}{c}\text { Cereclor } \\
\text { S45 }\end{array}$ & $\begin{array}{c}\text { Cereclor } \\
\text { 50LV }\end{array}$ & Huels 70C & CP-42 & CP-52 & $\begin{array}{c}\text { Overall } \\
\text { average }\end{array}$ \\
\hline Water samples & $144 \pm 43$ & $100 \pm 24$ & $89 \pm 19$ & $73 \pm 22$ & $88 \pm 15$ & 101 \\
\hline Daphnia samples & $172 \pm 57$ & $151 \pm 70$ & $99 \pm 26$ & $125 \pm 28$ & $139 \pm 37$ & 130 \\
\hline
\end{tabular}


Table S2. Comparison between modelled (mod.) and experimental (exp.) log BAF ( $\left.\mathrm{k} \mathrm{kg} \mathrm{lip}^{-1}\right)$ for the CP technical mixtures used in this study, as well as estimated rate constants for uptake through diet $\left(k \mathrm{~F}, \mathrm{~kg}\right.$ food $\mathrm{kg}$ organism day $\left.{ }^{-1}\right)$, uptake through respiration $\left(k_{1}, \mathrm{~L} \mathrm{~kg}\right.$ day $\left.^{-1}\right)$, elimination through respiration $\left(\mathrm{k}_{2}\right.$, day $\left.^{-1}\right)$, elimination through excretion of feces $\left(k_{\mathrm{E}}\right.$, day $\left.{ }^{-1}\right)$, growth $\left(k_{\mathrm{G}}\right.$, day $\left.{ }^{-1}\right)$ and metabolism $\left(k_{\mathrm{M}}\right.$, day $\left.^{-1}\right)$. Concentration of CPs in water $\left(\mathrm{C}_{\text {water }}(\exp ),. \mathrm{g} \mathrm{L}^{-1}\right)$ input values were obtained from this study, and used for the calculation of the concentration of CPs in D. magna originating from uptake through respiration $\left(\mathrm{C}_{\text {D.magna }}\right.$ (respiration), $\left.\mathrm{g} \mathrm{kg} \mathrm{ww}^{-1}\right)$. Concentration of $\mathrm{CPs}$ in D. magna originating from uptake through diet $\left(\mathrm{C}_{\text {D.magna }}(\mathrm{diet}), \mathrm{g} \mathrm{kg} \mathrm{ww}^{-1}\right)$ was estimated from the concentration of $\mathrm{CPs}_{\mathrm{s}}$ in algae, in turn estimated from the $K_{\text {oc-water }}$ calculated for these mixtures in a previous study ${ }^{3}$ and concentrations in water quantified in this study, using $50 \%$ organic carbon content (Table S3). CD.magna $\left(\mathrm{g} \mathrm{kg} \mathrm{ww}^{-1}\right.$ ) was calculated as according to KABAM version 1.0 (2009), from the different rate constants. Non-listed parameters were calculated as described in KABAM version 1.0 (2009). Other parameter values can be found in Table S4. References for the different values used are indicated within parenthesis.

\begin{tabular}{cccccccc} 
& Cereclor S45 & Cereclor 50LV & Huels 70C & CP-42 & CP-52 & PCB-138 & PCB-180 \\
\hline $\log$ BAF (exp.) & $\mathbf{6 . 7}$ & $\mathbf{6 . 9}$ & $\mathbf{7 . 0}$ & $\mathbf{6 . 5}$ & $\mathbf{7 . 0}$ & $\mathbf{7 . 3 0}^{(\mathbf{4})}$ & $\mathbf{7 . 2 5}^{(\mathbf{4})}$ \\
\hline $\log$ BAF (mod.) & $\mathbf{6 . 9}$ & $\mathbf{6 . 5}$ & $\mathbf{7 . 4}$ & $\mathbf{7 . 0}$ & $\mathbf{7 . 6}$ & $\mathbf{8 . 1}$ & $\mathbf{8 . 7}$ \\
\hline $\log K_{\text {ow }}$ & 7.88 & 6.24 & 7.03 & 9.79 & 8.07 & 6.83 & 7.36 \\
\hline $\mathrm{C}_{\text {water }}$ (exp.) & $1.2 \times 10^{-6}$ & $2.3 \times 10^{-6}$ & $5.8 \times 10^{-7}$ & $1.4 \times 10^{-6}$ & $1.4 \times 10^{-6}$ & $7.5 \times 10^{-13(4)}$ & $2.3 \times 10^{-13(5)}$ \\
\hline $\mathrm{C}_{\text {D.magna }}$ & 0.67 & 0.49 & 1.07 & 0.87 & 3.6 & $5.8 \times 10^{-6}$ & $8 \times 10^{-6}$ \\
\hline $\mathrm{C}_{\text {D.magna }}$ (diet) & 0.04 & 2.2 & 0.9 & 0.00057 & 0.37 & $7.6 \times 10^{-6}(5)$ & $3.5 \times 10^{-6}(5)$ \\
\hline $\mathrm{C}_{D \text {.magna }}$ (respiration) & 0.008 & 0.2 & 0.04 & 0.05 & 0.09 & $5 \times 10^{-8}$ & $1.5 \times 10^{-8}$ \\
\hline$k_{1}$ & $6.6 \times 10^{4}$ & $6.6 \times 10^{4}$ & $6.6 \times 10^{4}$ & $6.6 \times 10^{4}$ & $6.6 \times 10^{4}$ & $6.6 \times 10^{4}$ & $6.6 \times 10^{4}$ \\
\hline$k_{\mathrm{F}}$ & 0.12 & 1.2 & 0.6 & 0.002 & 0.08 & 0.76 & 0.34 \\
\hline$k_{2}$ & 0.11 & 4.7 & 0.76 & 0.001 & 0.07 & 1.2 \\
\hline$k_{\mathrm{E}}$ & 0.01 & 0.10 & 0.05 & 0.00014 & 0.007 & 0.06 & 0.36 \\
\hline$k_{\mathrm{G}}$ & 0.04 & 0.04 & 0.04 & 0.04 & 0.04 & 0.04
\end{tabular}




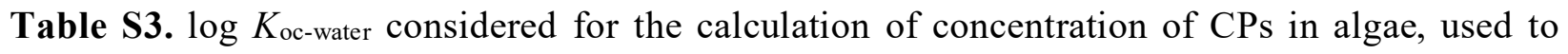
estimate the concentration of CPs in D. magna originating from uptake through diet $\left(\mathrm{C}_{\text {D.magna }}\right.$ (diet), $\mathrm{g} \mathrm{kg} \mathrm{ww}^{-1}$ ) in Table S2. References for the different values used are indicated within parenthesis.

\begin{tabular}{rcll} 
CP substance & $\log$ Koc & $\mathbf{C}_{\text {algae }}(\mathbf{g} / \mathbf{k g w w})$ & $\mathbf{C}_{\text {D.magna }}(\mathbf{d i e t})$ \\
\hline Cereclor S45 & $5.73^{(3)}$ & 0.29 & 0.04 \\
\hline Cereclor 50LV & $6.24^{(3)}$ & 1.8 & 2.2 \\
\hline Huels 70C & $6.76^{(3)}$ & 1.5 & 0.9 \\
\hline CP-42 & $5.73^{*}$ & 0.34 & 0.00057 \\
\hline CP-52 & $6.3^{(3)}$ & 2.1 & 0.37 \\
\hline PCB-138 & - & $1.0 \times 10^{-5(5)}$ & $7.6 \times 10^{-6}$ \\
\hline PCB-180 & - & $1.0 \times 10^{-5(5)}$ & $3.6 \times 10^{-6}$ \\
\hline
\end{tabular}

*not measured in Castro et al., 2018, assumed to be closest to that of Cereclor S45 
Table S4. Values used for the different physical-chemical and biological parameters used in the KABAM model, necessary to most accurately model log BAF (L kg lip ${ }^{-1}$, Table S2).

\begin{tabular}{rcll} 
Parameter & Value & Unit & Reference \\
\hline \hline Temperature & 20 & ${ }^{\circ} \mathrm{C}$ & Standard \\
\hline \hline Total organic carbon in water & $2 \times 10^{-9}$ & $\mathrm{~kg} / \mathrm{L}$ & Measured \\
\hline \hline Fraction of diet containing the chemical & 100 & $\%$ & \\
\hline Algae cell dry weight & $1.42 \times 10^{-14}$ & $\mathrm{~kg}$ & 6 \\
\hline Algae cell water content & 94.64 & $\%$ & $7 *$ \\
\hline Algae cell wet weight & $2.7 \times 10^{-13}$ & $\mathrm{~kg}$ & 6,7 \\
\hline Algae cell organic carbon $(\mathrm{dw})$ & 50 & $\%$ & \\
\hline Algae cell organic carbon & $7.1 \times 10^{-15}$ & $\mathrm{~kg}$ & \\
\hline D. magna dry weight & $2.5 \times 10^{-8}$ & $\mathrm{~kg}$ & This work ${ }^{* *}$ \\
\hline D. magna water content & 90 & $\%$ & This work ${ }^{* *}$ \\
\hline D. magna wet weight & $2.5 \times 10^{-7}$ & $\mathrm{~kg}$ & This work** \\
\hline D. magna lipid content $(\mathrm{dw})$ & 7 & $\%$ & This work** \\
\hline D. magna lipid content & $1.75 \times 10^{-8}$ & $\mathrm{~kg}$ & This work** \\
\hline D. magna organic carbon $(\mathrm{dw})$ & 38.5 & $\%$ & 3 \\
\hline D. magna organic carbon & $9.6 \times 10^{-7}$ & $\mathrm{~kg}$ & 3 \\
\hline & & & \\
\hline
\end{tabular}

*value for Desmodesmus sp., same order as P.subcapitata.

** values obtained for a fed juvenile D. magna (3-4 days old), as used in this study. 


\section{References}

(1) Arnot, J. A.; Gobas, F. A. P. C. A food web bioaccumulation model for organic chemicals in aquatic ecosystems. Environ. Toxicol. Chem. 2004, 23 (10), 2343-2355.

(2) Barata, C.; Alañon, P.; Gutierrez-Alonso, S.; Riva, M. C.; Fernández, C.; Tarazona, J. V. A Daphnia magna feeding bioassay as a cost effective and ecological relevant sublethal toxicity test for Environmental Risk Assessment of toxic effluents. Sci. Total Environ. 2008.

(3) Castro, M.; Breitholtz, M.; Yuan, B.; Athanassiadis, I.; Asplund, L.; Sobek, A. Partitioning of Chlorinated Paraffins (CPs) to Daphnia magna Overlaps between Restricted and in-Use Categories. Environ. Sci. Technol. 2018, 52 (17), 9713-9721.

(4) Sobek, A.; McLachlan, M. S.; Borgå, K.; Asplund, L.; Lundstedt-Enkel, K.; Polder, A.; Gustafsson $\mathrm{O}$, Ö. A comparison of PCB bioaccumulation factors between an arctic and a temperate marine food web. Sci. Total Environ. 2010, 408 (13), 2753-2760.

(5) Tiano, M.; Tronczyński, J.; Harmelin-Vivien, M.; Tixier, C.; Carlotti, F. PCB concentrations in plankton size classes, a temporal study in Marseille Bay, Western Mediterranean Sea. Mar. Pollut. Bull. 2014.

(6) Bossuyt, B. T. A.; Janssen, C. R. Long-term acclimation of Pseudokirchneriella subcapitata (Korshikov) Hindak to different copper concentrations: Changes in tolerance and physiology. Aquat. Toxicol. 2004.

(7) Li, Z.; Li, Y.; Zhang, X.; Tan, T. Lipid extraction from non-broken and high water content microalgae Chlorella spp. by three-phase partitioning. Algal Res. 2015. 
Figure S1. Equilibration time for CP-52 in D.magna, using passive dosing. Concentrations of CP$52\left(\mathrm{ng} \mu \mathrm{g} \mathrm{dw}^{-1}\right)$ were measured after 24, 48 and 72 hours of contact time between dosed silicone, dosed water and D. magna ( $<3$ days old).

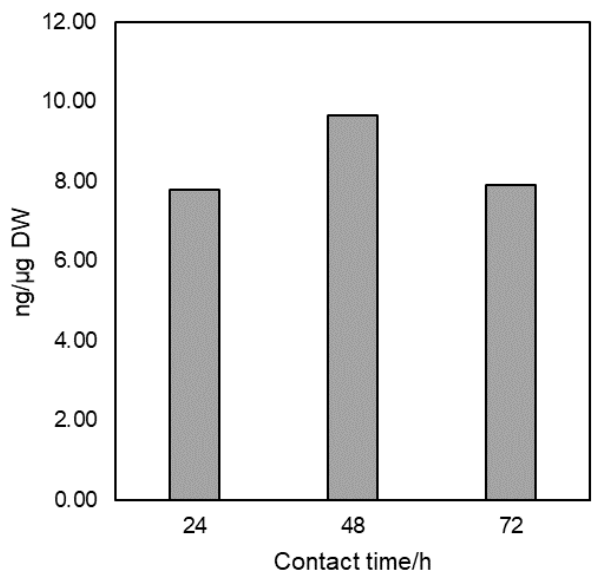


Figure S2. Depuration expressed as decrease in concentration in D. magna (compared to initial concentration at $\left.\mathrm{t}=0 ; \mathrm{C}_{\text {D.magna }}\right)$ versus time $(t)$, based on the calculated first order kinetic depuration rate constants for Cereclor 50LV (A), Huels 70C (B), Cereclor S45 (C), CP-52 (D) and CP-42 (E) with clean food and water (yellow, $\mathbf{\Delta}$ ) vs no food, just clean water (blue, $\bullet$ ).

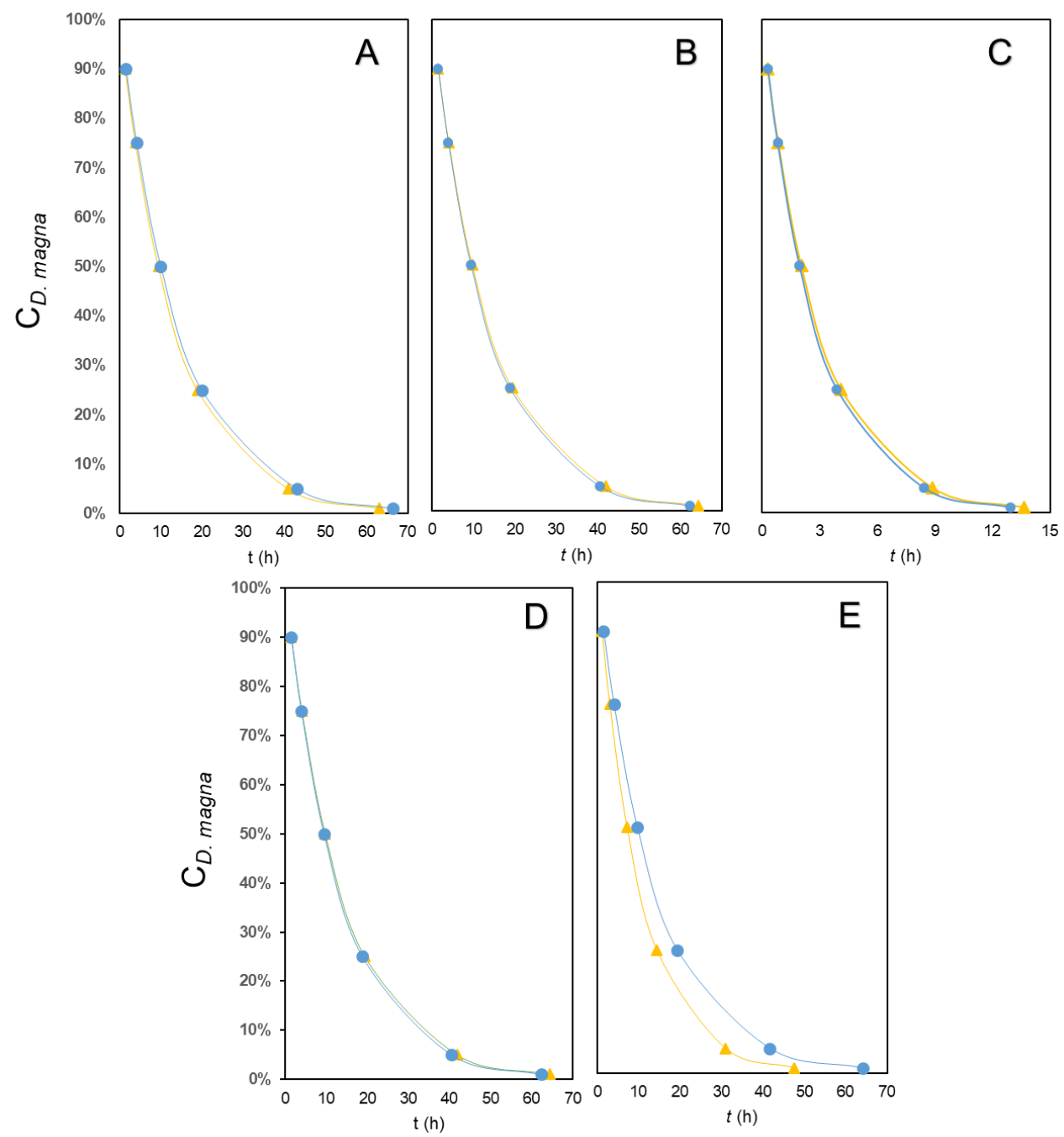


Figure S3. Visualization of the depuration progress calculated from the depuration rate constant (y axis, \% $\mathrm{C}_{\text {D.magna }}$ ) for the five different $\mathrm{CP}$ technical substances in $D$. magna over time (x axis, $t$

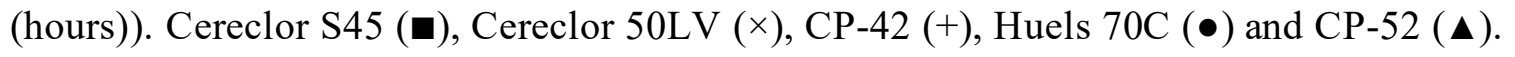

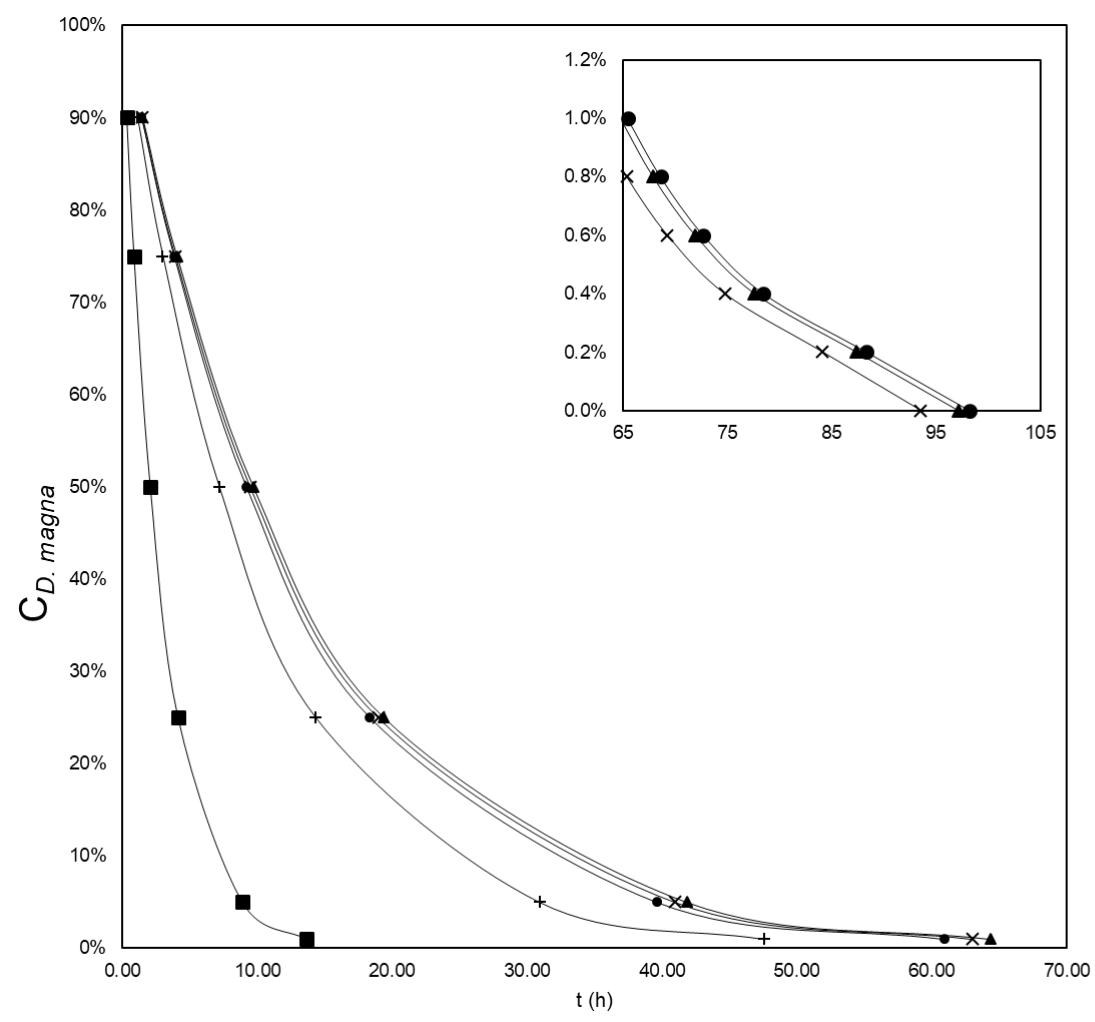


Table S5. Generalized linear models testing effects of the different CP mixtures on $\log \mathrm{BCF}$, using Cereclor 50LV and Huels 70C (SCCPs) as references for comparison.

\begin{tabular}{ccccc} 
Cereclor 50LV vs & Estimate & SE & t-value & p-value \\
\hline \hline CP-42 & -0.06011 & 0.021 & -2.865 & 0.00832 \\
\hline Cereclor S45 & -0.03113 & 0.021 & -1.495 & 0.14749 \\
\hline CP-52 & -0.00494 & 0.021 & -0.237 & 0.815 \\
\hline Huels 70C & 0.01812 & 0.021 & 0.881 & 0.38679 \\
\hline Huels 70C vs & & & & \\
\hline CP-42 & -0.07823 & 0.021 & -3.745 & 0.000950 \\
\hline Cereclor S45 & -0.04925 & 0.021 & -2.375 & 0.025516 \\
\hline Cereclor 50LV & -0.01812 & 0.021 & -0.881 & 0.386788 \\
\hline CP-52 & -0.0231 & 0.021 & -1.110 & 0.27738 \\
\hline
\end{tabular}


Table S6. Generalized linear models testing effects of the different CP mixtures on log BAF, using Cereclor 50LV and Huels 70C (SCCPs) as references for comparison.

\begin{tabular}{ccccc} 
Cereclor 50LV vs & Estimate & SE & t-value & p-value \\
\hline \hline CP-42 & -0.047326 & 0.011 & -4.260 & 0.000254 \\
\hline Cereclor S45 & -0.019306 & 0.011 & -1.750 & 0.092377 \\
\hline CP-52 & 0.0214 & 0.011 & 1.966 & 0.061 \\
\hline Huels 70C & 0.034898 & 0.011 & 3.206 & 0.003657 \\
\hline Huels 70C vs & & & & \\
\hline CP-42 & -0.082224 & 0.011 & -7.463 & $8.13 \mathrm{e}-08$ \\
\hline Cereclor S45 & -0.054203 & 0.011 & -4.956 & $4.18 \mathrm{e}-05$ \\
\hline Cereclor 50LV & -0.034898 & 0.011 & -3.206 & 0.0037 \\
\hline CP-52 & -0.0135 & 0.011 & -1.253 & 0.222 \\
\hline
\end{tabular}


Table S7. Two sample t-test comparison of the $\log \mathrm{BCF}$ and $\log \mathrm{BAF}$ obtained for the different CP technical mixtures.

\begin{tabular}{cccc} 
& t-value & df & p-value \\
\hline \hline Cereclor S45 & 3.2785 & 5 & 0.02199 \\
\hline Cereclor 50LV & 5.1325 & 5 & 0.003668 \\
\hline Huels 70C & 1.9095 & 5 & 0.1145 \\
\hline CP-42 & 2.7683 & 5 & 0.03945 \\
\hline CP-52 & 3.7928 & 5 & 0.0127 \\
\hline
\end{tabular}


Table S8. Linear regressions $(y=a x+b)$ for the relationships between chlorine content and between $\log \mathrm{BCF}$ and BAF. The p-value describes how good chlorine is as a predictor for the response variables (log $\mathrm{BCF}$ and $\log \mathrm{BAF})$.

\begin{tabular}{cccccc} 
& $\mathrm{a}$ & $\mathrm{b}$ & $\mathrm{df}$ & $\mathrm{R}^{2}$ & $\mathrm{p}$ \\
\hline \hline $\log \mathrm{BCF}$ & 0.0167 & 0.005 & 28 & 0.27 & 0.00215 \\
\hline $\log \mathrm{BAF}$ & 0.0177 & 0.003 & 28 & 0.54 & $2.06 \times 10^{-6}$ \\
\hline
\end{tabular}


Table S9. Generalized linear models testing effects using CP-42 as reference the different log BCF and BAF values obtained for the different substances, used for Figure 2.

\section{BCF comparison}

\begin{tabular}{ccccc} 
CP-42 vs & Estimate & SE & t-value & p-value \\
\hline Cereclor S45 & 0.029 & 0.21 & 1.360 & 0.186 \\
\hline Cereclor 50LV & 0.06 & 0.2115 & 2.842 & 0.00879 \\
\hline CP-52 & 0.0552 & 0.0212 & 2.606 & 0.01523 \\
\hline Huels 70C & 0.07823 & 0.0211 & 3.715 & 0.00103 \\
\hline
\end{tabular}

BAF comparison

\begin{tabular}{ccccc} 
CP-42 vs & & & \\
\hline \hline Cereclor S45 & 0.028 & 0.0111 & 2.520 & 0.019 \\
\hline Cereclor 50LV & 0.047 & 0.0111 & 4.276 & 0.000243 \\
\hline CP-52 & 0.0687 & 0.0110 & 6.240 & $1.58 \times 10^{-6}$ \\
\hline Huels 70C & 0.0822 & 0.0110 & 7.492 & $7.61 \times 10^{-8}$ \\
\hline
\end{tabular}


Figure S4. Congener pattern observed in D. magna after Cereclor 50LV aqueous exposure (top) and original CP pattern of Cereclor 50LV (bottom). Similarity between patterns was $>84$ using the goodness of fit $\left(\mathrm{R}^{2}\right)$ between the pattern of the technical mixture and that of the sample.

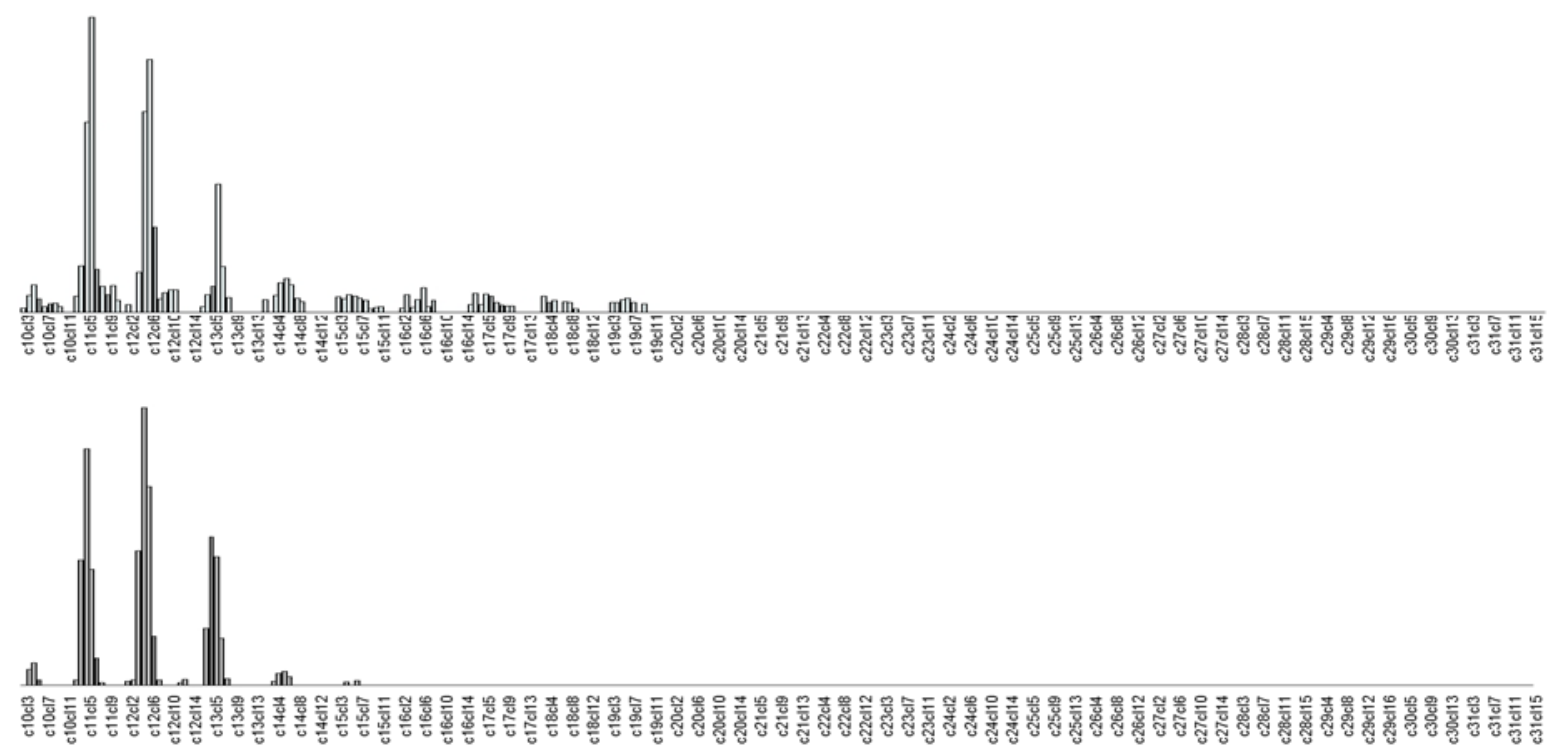


Figure S5. Congener pattern observed in D. magna after Huels 70C aqueous exposure (top) and original CP pattern of Huels 70C (bottom). Similarity between patterns was $>95$ using the goodness of fit $\left(\mathrm{R}^{2}\right)$ between the pattern of the technical mixture and that of the sample.
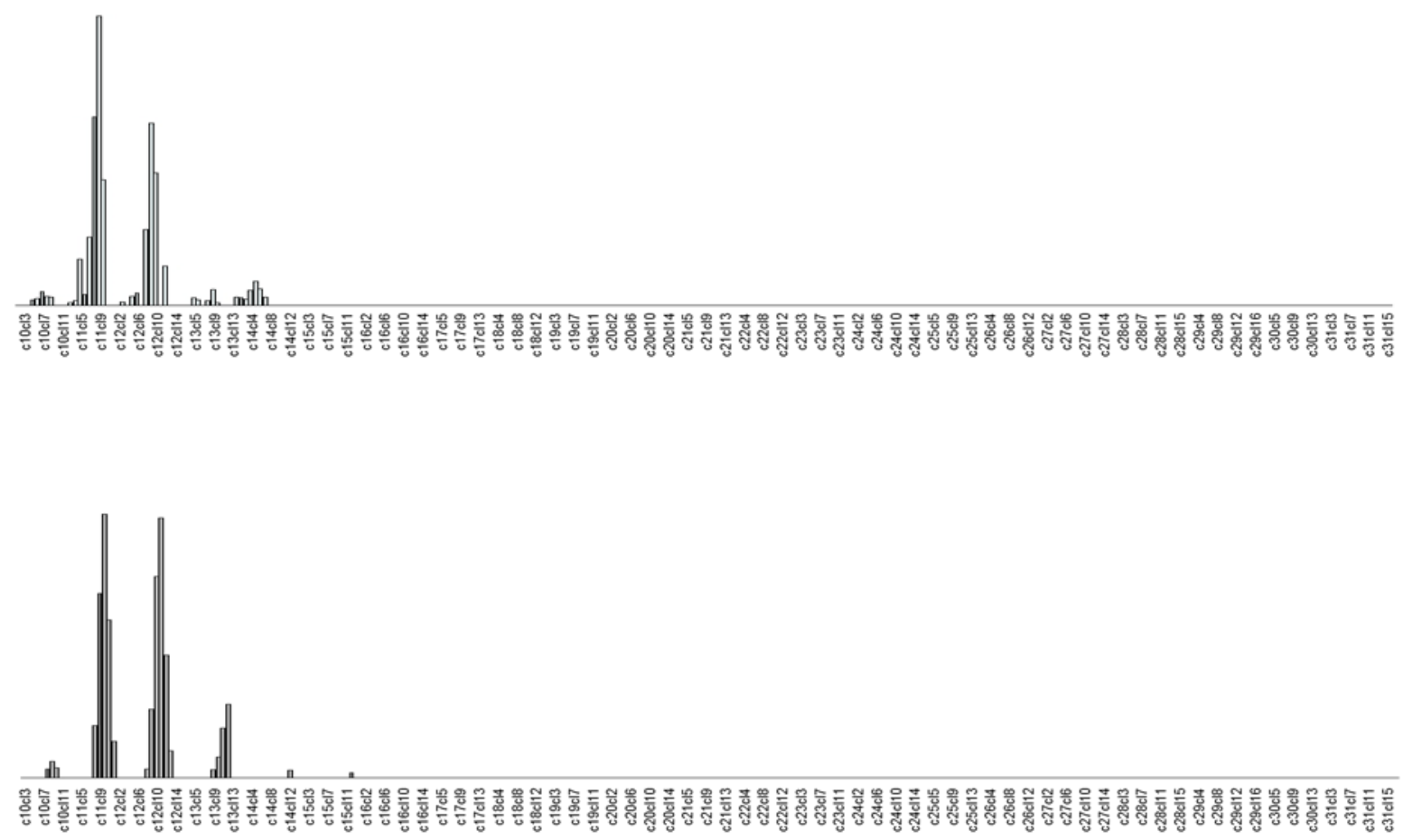
Figure S6. Congener pattern observed in D. magna after Cereclor S45 aqueous exposure (top) and original CP pattern of Cereclor S45 (bottom). Similarity between patterns was $>90$ using the goodness of fit $\left(\mathrm{R}^{2}\right)$ between the pattern of the technical mixture and that of the sample.
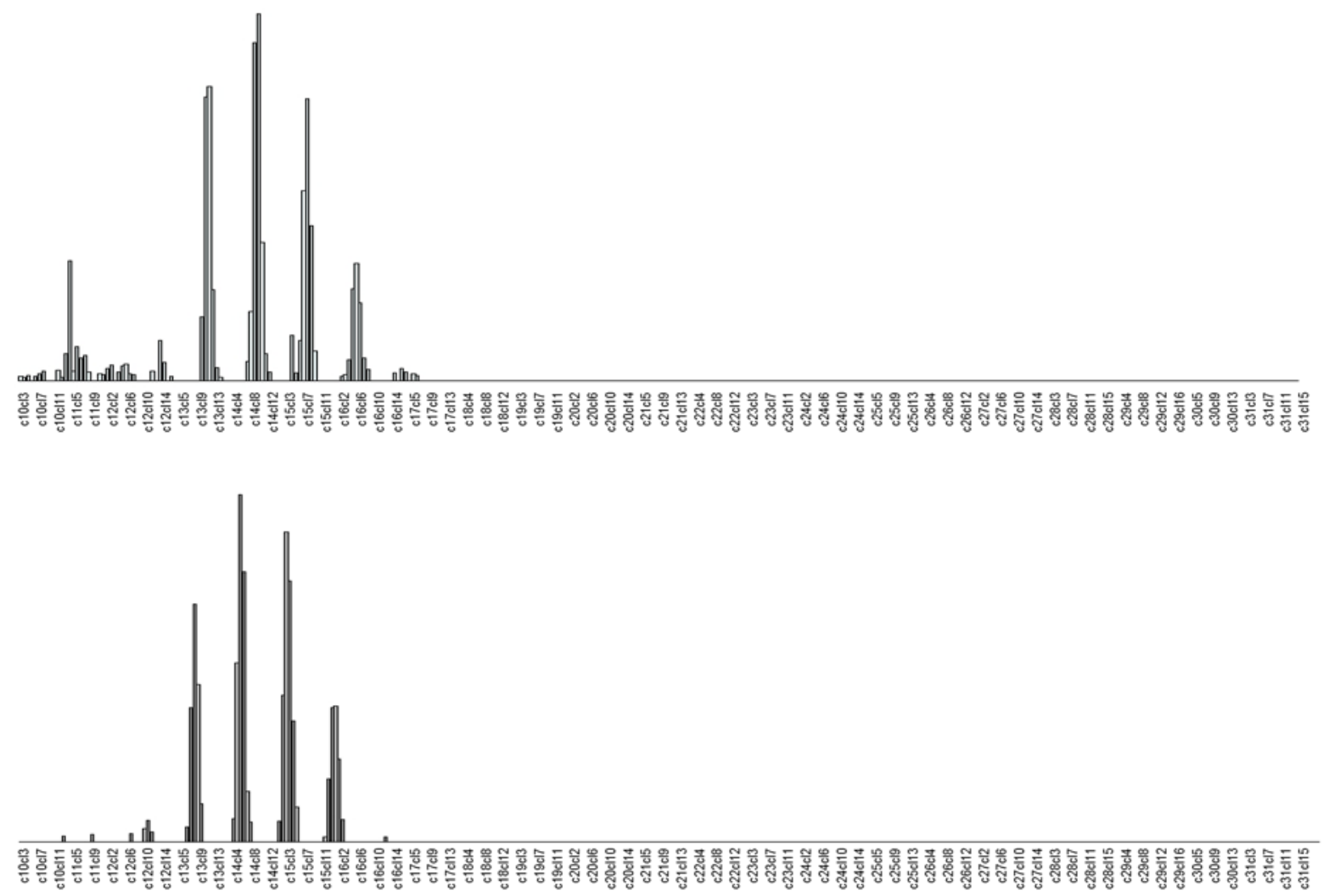
Figure S7. Congener pattern observed in D. magna after CP-52 aqueous exposure (bottom) and original CP pattern of CP-52 (top). Similarity between patterns was $>80$ using the goodness of fit $\left(\mathrm{R}^{2}\right)$ between the pattern of the technical mixture and that of the sample.
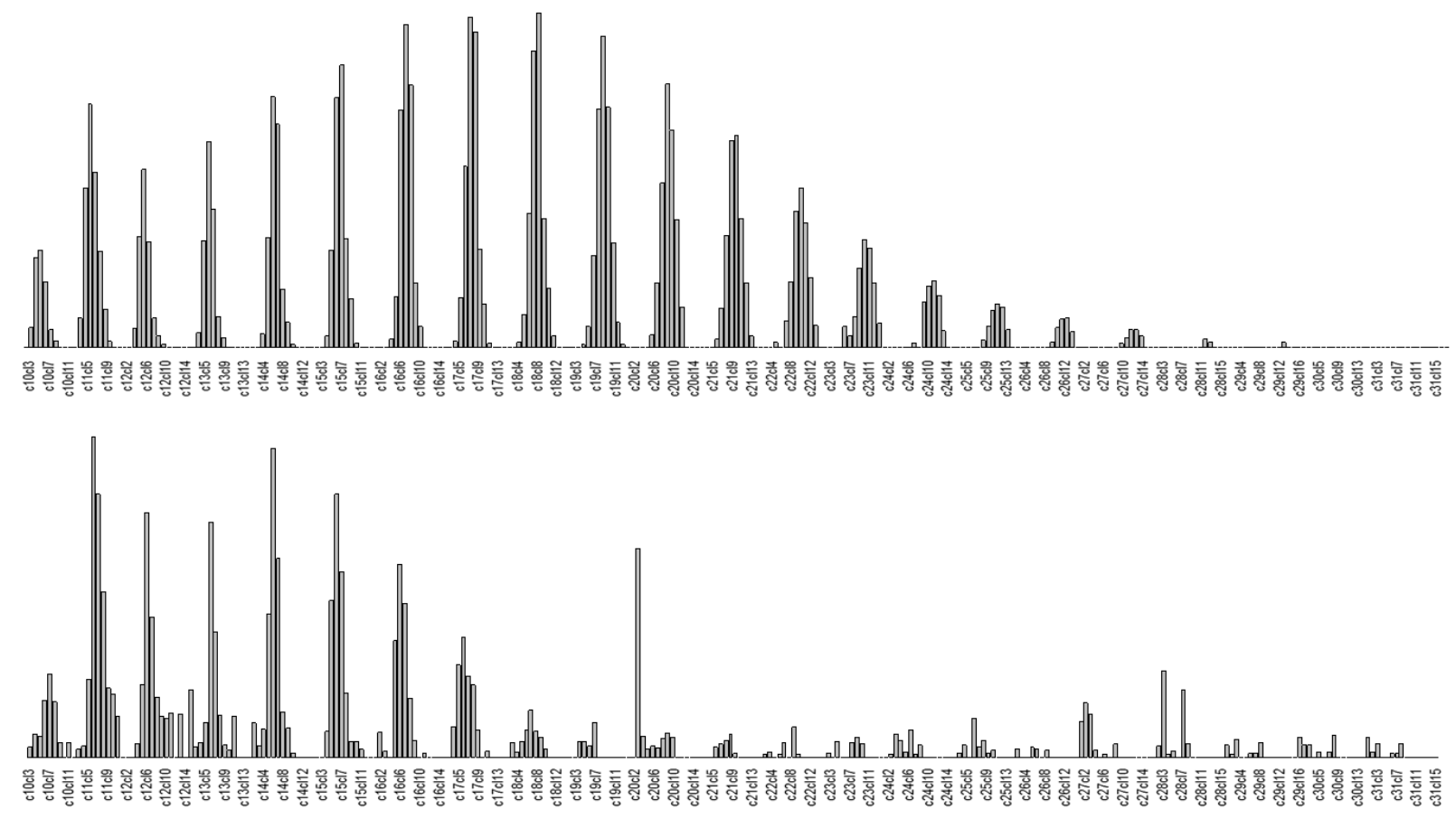
Table S10. List of congeners with molecular weight above $650 \mathrm{Da}$ found in the original CP technical mixture and in exposed D. magna.

\begin{tabular}{|c|c|c|c|c|c|c|}
\hline & $\mathrm{Cl} \%$ & MW & CP-42 & D. magna & CP-52 & D. magna \\
\hline $\mathrm{C}_{18} \mathrm{Cl}_{12}$ & 64 & 667.84 & $\mathrm{x}$ & - & $\mathrm{x}$ & - \\
\hline $\mathrm{C}_{19} \mathrm{Cl}_{12}$ & 62 & 681.86 & $\mathrm{x}$ & - & $\mathrm{x}$ & - \\
\hline $\mathrm{C}_{20} \mathrm{Cl}_{11}$ & 59 & 661.44 & $\mathrm{x}$ & - & $\mathrm{x}$ & $\mathrm{x}$ \\
\hline $\mathrm{C}_{20} \mathrm{Cl}_{12}$ & 61 & 695.89 & - & - & $\mathrm{x}$ & - \\
\hline $\mathrm{C}_{21} \mathrm{Cl}_{10}$ & 55 & 641.02 & - & - & $\mathrm{x}$ & $\mathrm{x}$ \\
\hline $\mathrm{C}_{21} \mathrm{Cl}_{11}$ & 58 & 675.47 & - & - & $\mathrm{x}$ & $\mathrm{x}$ \\
\hline $\mathrm{C}_{21} \mathrm{Cl}_{12}$ & 60 & 709.91 & - & - & $\mathrm{x}$ & - \\
\hline $\mathrm{C}_{22} \mathrm{Cl}_{10}$ & 54 & 655.05 & $\mathrm{x}$ & $\mathrm{x}$ & $\mathrm{x}$ & $\mathrm{x}$ \\
\hline $\mathrm{C}_{22} \mathrm{Cl}_{11}$ & 57 & 689.50 & $\mathrm{x}$ & - & $\mathrm{x}$ & $\mathrm{x}$ \\
\hline $\mathrm{C}_{22} \mathrm{Cl}_{12}$ & 59 & 723.94 & - & - & $\mathrm{x}$ & - \\
\hline $\mathrm{C}_{23} \mathrm{Cl}_{10}$ & 53 & 669.08 & $\mathrm{x}$ & $\mathrm{x}$ & $\mathrm{x}$ & $\mathrm{x}$ \\
\hline $\mathrm{C}_{23} \mathrm{Cl}_{11}$ & 55 & 703.52 & - & - & $\mathrm{x}$ & $\mathrm{x}$ \\
\hline $\mathrm{C}_{23} \mathrm{Cl}_{12}$ & 58 & 737.97 & - & - & $\mathrm{x}$ & - \\
\hline $\mathrm{C}_{24} \mathrm{Cl}_{10}$ & 52 & 683.10 & $\mathrm{x}$ & $\mathrm{x}$ & $\mathrm{x}$ & $\mathrm{x}$ \\
\hline $\mathrm{C}_{24} \mathrm{Cl}_{11}$ & 54 & 717.55 & $\mathrm{x}$ & $\mathrm{x}$ & $\mathrm{x}$ & $\mathrm{x}$ \\
\hline $\mathrm{C}_{24} \mathrm{Cl}_{12}$ & 57 & 751.99 & $\mathrm{x}$ & - & $\mathrm{x}$ & - \\
\hline $\mathrm{C}_{25} \mathrm{Cl}_{9}$ & 48 & 662.69 & $\mathrm{x}$ & $\mathrm{x}$ & $\mathrm{x}$ & $\mathrm{x}$ \\
\hline $\mathrm{C}_{25} \mathrm{Cl}_{10}$ & 51 & 697.13 & $\mathrm{x}$ & $\mathrm{x}$ & $\mathrm{x}$ & $\mathrm{x}$ \\
\hline $\mathrm{C}_{25} \mathrm{Cl}_{11}$ & 53 & 731.58 & $\mathrm{x}$ & $\mathrm{x}$ & $\mathrm{x}$ & $\mathrm{x}$ \\
\hline $\mathrm{C}_{25} \mathrm{Cl}_{12}$ & 56 & 766.02 & $\mathrm{x}$ & - & $\mathrm{x}$ & - \\
\hline $\mathrm{C}_{26} \mathrm{Cl}_{9}$ & 47 & 676.71 & $\mathrm{x}$ & $\mathrm{x}$ & $\mathrm{x}$ & $\mathrm{x}$ \\
\hline $\mathrm{C}_{26} \mathrm{Cl}_{10}$ & 50 & 711.16 & $\mathrm{x}$ & $\mathrm{x}$ & $\mathrm{x}$ & $\mathrm{x}$ \\
\hline $\mathrm{C}_{26} \mathrm{Cl}_{11}$ & 52 & 745.60 & $\mathrm{x}$ & $\mathrm{x}$ & $\mathrm{x}$ & $x$ \\
\hline $\mathrm{C}_{26} \mathrm{Cl}_{12}$ & 55 & 780.05 & $\mathrm{x}$ & - & $\mathrm{x}$ & - \\
\hline $\mathrm{C}_{27} \mathrm{Cl}_{8}$ & 43 & 656.29 & $\mathrm{x}$ & $\mathrm{x}$ & $\mathrm{x}$ & $\mathrm{x}$ \\
\hline $\mathrm{C}_{27} \mathrm{Cl}_{9}$ & 46 & 690.74 & $\mathrm{x}$ & $\mathrm{x}$ & $\mathrm{x}$ & $\mathrm{x}$ \\
\hline $\mathrm{C}_{27} \mathrm{Cl}_{10}$ & 49 & 725.18 & $\mathrm{x}$ & $\mathrm{x}$ & $\mathrm{x}$ & $\mathrm{x}$ \\
\hline $\mathrm{C}_{27} \mathrm{Cl}_{11}$ & 51 & 759.63 & $\mathrm{x}$ & $\mathrm{x}$ & $\mathrm{x}$ & $\mathrm{x}$ \\
\hline $\mathrm{C}_{27} \mathrm{Cl}_{12}$ & 54 & 794.07 & $\mathrm{x}$ & - & $\mathrm{x}$ & - \\
\hline $\mathrm{C}_{28} \mathrm{Cl}_{8}$ & 42 & 670.32 & $\mathrm{x}$ & $\mathrm{x}$ & $\mathrm{x}$ & $\mathrm{x}$ \\
\hline $\mathrm{C}_{28} \mathrm{Cl}_{9}$ & 45 & 704.77 & $\mathrm{x}$ & $\mathrm{x}$ & $\mathrm{x}$ & $\mathrm{x}$ \\
\hline $\mathrm{C}_{28} \mathrm{Cl}_{10}$ & 48 & 739.21 & $\mathrm{x}$ & $\mathrm{x}$ & $\mathrm{x}$ & $\mathrm{x}$ \\
\hline $\mathrm{C}_{28} \mathrm{Cl}_{11}$ & 50 & 773.66 & $\mathrm{x}$ & $\mathrm{x}$ & $\mathrm{x}$ & $\mathrm{x}$ \\
\hline $\mathrm{C}_{28} \mathrm{Cl}_{12}$ & 53 & 808.10 & $\mathrm{x}$ & - & $\mathrm{x}$ & - \\
\hline $\mathrm{C}_{29} \mathrm{Cl}_{8}$ & 41 & 684.35 & $\mathrm{x}$ & $\mathrm{x}$ & $\mathrm{x}$ & $\mathrm{x}$ \\
\hline $\mathrm{C}_{29} \mathrm{Cl}_{9}$ & 44 & 718.79 & $\mathrm{x}$ & $\mathrm{x}$ & $\mathrm{x}$ & $\mathrm{x}$ \\
\hline $\mathrm{C}_{29} \mathrm{Cl}_{10}$ & 47 & 753.24 & $\mathrm{x}$ & $\mathrm{x}$ & $\mathrm{x}$ & $\mathrm{x}$ \\
\hline
\end{tabular}




\begin{tabular}{|c|c|c|c|c|c|c|}
\hline $\mathrm{C}_{29} \mathrm{Cl}_{11}$ & 50 & 787.68 & $\mathrm{X}$ & $\mathrm{X}$ & $\mathrm{X}$ & $\mathrm{X}$ \\
\hline $\mathrm{C}_{29} \mathrm{Cl}_{12}$ & 52 & 822.13 & $\mathrm{X}$ & - & $\mathrm{X}$ & - \\
\hline $\mathrm{C}_{30} \mathrm{Cl}_{7}$ & 37 & 663.93 & $\mathrm{x}$ & $\mathrm{X}$ & $\mathrm{X}$ & $\mathrm{X}$ \\
\hline $\mathrm{C}_{30} \mathrm{Cl}_{8}$ & 41 & 698.37 & $\mathrm{x}$ & $\mathrm{x}$ & $\mathrm{X}$ & $\mathrm{X}$ \\
\hline $\mathrm{C}_{30} \mathrm{Cl}_{9}$ & 44 & 732.82 & $\mathrm{X}$ & $\mathrm{x}$ & $\mathrm{X}$ & $\mathrm{x}$ \\
\hline $\mathrm{C}_{30} \mathrm{Cl}_{10}$ & 46 & 767.26 & $\mathrm{X}$ & $\mathrm{X}$ & $\mathrm{X}$ & $\mathrm{X}$ \\
\hline $\mathrm{C}_{30} \mathrm{Cl}_{11}$ & 49 & 801.71 & $\mathrm{X}$ & - & $\mathrm{X}$ & $\mathrm{x}$ \\
\hline $\mathrm{C}_{30} \mathrm{Cl}_{12}$ & 51 & 836.15 & - & - & $\mathrm{X}$ & $\mathrm{x}$ \\
\hline $\mathrm{C}_{31} \mathrm{Cl}_{7}$ & 37 & 677.96 & $\mathrm{X}$ & $\mathrm{X}$ & $\mathrm{X}$ & $\mathrm{x}$ \\
\hline $\mathrm{C}_{31} \mathrm{Cl}_{8}$ & 40 & 712.40 & $\mathrm{x}$ & $\mathrm{X}$ & $\mathrm{X}$ & $\mathrm{x}$ \\
\hline $\mathrm{C}_{31} \mathrm{Cl}_{9}$ & 43 & 746.85 & $\mathrm{x}$ & $\mathrm{X}$ & $\mathrm{X}$ & $\mathrm{X}$ \\
\hline $\mathrm{C}_{31} \mathrm{Cl}_{10}$ & 45 & 781.29 & $\mathrm{x}$ & $\mathrm{X}$ & $\mathrm{X}$ & $\mathrm{X}$ \\
\hline $\mathrm{C}_{31} \mathrm{Cl}_{11}$ & 48 & 815.74 & $\mathrm{x}$ & $\mathrm{X}$ & $\mathrm{x}$ & - \\
\hline $\mathrm{C}_{31} \mathrm{Cl}_{12}$ & 50 & 850.18 & $\mathrm{x}$ & - & $\mathrm{x}$ & - \\
\hline
\end{tabular}


Table S11. List of congeners with $\log K_{\text {ow }}$ above 12 found originally in the $\mathrm{CP}$ technical mixture and in exposed D. magna.

\begin{tabular}{|c|c|c|c|c|c|c|}
\hline & $\mathrm{Cl} \%$ & $\log K_{\text {ow }}$ & CP-42 & D. magna & CP-52 & D. magna \\
\hline $\mathrm{C}_{22} \mathrm{Cl}_{5}$ & 37 & 12.05 & $\mathrm{x}$ & $\mathrm{x}$ & $\mathrm{x}$ & $\mathrm{x}$ \\
\hline $\mathrm{C}_{22} \mathrm{Cl}_{6}$ & 41 & 12.23 & $\mathrm{x}$ & $\mathrm{x}$ & $\mathrm{x}$ & $\mathrm{x}$ \\
\hline $\mathrm{C}_{22} \mathrm{Cl}_{7}$ & 45 & 12.41 & $\mathrm{x}$ & $\mathrm{x}$ & $x$ & $\mathrm{x}$ \\
\hline $\mathrm{C}_{22} \mathrm{Cl}_{8}$ & 48 & 12.59 & $\mathrm{x}$ & $\mathrm{x}$ & $x$ & $\mathrm{x}$ \\
\hline $\mathrm{C}_{22} \mathrm{Cl}_{9}$ & 51 & 12.77 & $\mathrm{x}$ & $\mathrm{x}$ & $x$ & $\mathrm{x}$ \\
\hline $\mathrm{C}_{22} \mathrm{Cl}_{10}$ & 54 & 12.95 & $\mathrm{x}$ & - & $\mathrm{x}$ & - \\
\hline $\mathrm{C}_{22} \mathrm{Cl}_{11}$ & 57 & 13.13 & $\mathrm{x}$ & - & $x$ & - \\
\hline $\mathrm{C}_{22} \mathrm{Cl}_{12}$ & 59 & 13.31 & $\mathrm{x}$ & - & $x$ & - \\
\hline $\mathrm{C}_{23} \mathrm{Cl}_{2}$ & 18 & 12.00 & $\mathrm{x}$ & - & $x$ & - \\
\hline $\mathrm{C}_{23} \mathrm{Cl}_{3}$ & 25 & 12.18 & $\mathrm{x}$ & - & $x$ & - \\
\hline $\mathrm{C}_{23} \mathrm{Cl}_{4}$ & 31 & 12.36 & $\mathrm{x}$ & - & $x$ & $\mathrm{x}$ \\
\hline $\mathrm{C}_{23} \mathrm{Cl}_{5}$ & 36 & 12.54 & $\mathrm{x}$ & $\mathrm{x}$ & $\mathrm{x}$ & $\mathrm{x}$ \\
\hline $\mathrm{C}_{23} \mathrm{Cl}_{6}$ & 40 & 12.72 & $\mathrm{x}$ & $\mathrm{x}$ & $\mathrm{x}$ & $\mathrm{x}$ \\
\hline $\mathrm{C}_{23} \mathrm{Cl}_{7}$ & 44 & 12.9 & $\mathrm{x}$ & $\mathrm{x}$ & $\mathrm{x}$ & $\mathrm{x}$ \\
\hline $\mathrm{C}_{23} \mathrm{Cl}_{8}$ & 47 & 12.59 & $\mathrm{x}$ & $\mathrm{x}$ & $\mathrm{x}$ & $\mathrm{x}$ \\
\hline $\mathrm{C}_{23} \mathrm{Cl}_{9}$ & 50 & 13.26 & $\mathrm{x}$ & $\mathrm{x}$ & $\mathrm{x}$ & $\mathrm{x}$ \\
\hline $\mathrm{C}_{23} \mathrm{Cl}_{10}$ & 53 & 13.44 & $\mathrm{x}$ & $\mathrm{x}$ & $\mathrm{x}$ & $\mathrm{x}$ \\
\hline $\mathrm{C}_{23} \mathrm{Cl}_{11}$ & 55 & 13.62 & - & - & $\mathrm{x}$ & - \\
\hline $\mathrm{C}_{23} \mathrm{Cl}_{12}$ & 58 & 13.8 & - & - & $\mathrm{x}$ & - \\
\hline $\mathrm{C}_{24} \mathrm{Cl}_{2}$ & 17 & 12.49 & $\mathrm{x}$ & - & $\mathrm{x}$ & - \\
\hline $\mathrm{C}_{24} \mathrm{Cl}_{3}$ & 24 & 12.67 & $\mathrm{x}$ & - & $\mathrm{x}$ & $\mathrm{x}$ \\
\hline $\mathrm{C}_{24} \mathrm{Cl}_{4}$ & 30 & 12.85 & $\mathrm{x}$ & $\mathrm{x}$ & $\mathrm{x}$ & $\mathrm{x}$ \\
\hline $\mathrm{C}_{24} \mathrm{Cl}_{5}$ & 35 & 13.03 & $\mathrm{x}$ & $\mathrm{x}$ & $\mathrm{x}$ & $\mathrm{x}$ \\
\hline $\mathrm{C}_{24} \mathrm{Cl}_{6}$ & 39 & 13.21 & $\mathrm{x}$ & $\mathrm{x}$ & $\mathrm{x}$ & $\mathrm{x}$ \\
\hline $\mathrm{C}_{24} \mathrm{Cl}_{7}$ & 43 & 13.39 & $\mathrm{x}$ & $\mathrm{x}$ & $\mathrm{x}$ & $\mathrm{x}$ \\
\hline $\mathrm{C}_{24} \mathrm{Cl}_{8}$ & 46 & 13.57 & $\mathrm{x}$ & $\mathrm{x}$ & $\mathrm{x}$ & $\mathrm{x}$ \\
\hline $\mathrm{C}_{24} \mathrm{Cl}_{9}$ & 49 & 13.75 & $\mathrm{x}$ & $\mathrm{x}$ & $\mathrm{x}$ & $\mathrm{x}$ \\
\hline $\mathrm{C}_{24} \mathrm{Cl}_{10}$ & 52 & 13.93 & $\mathrm{x}$ & $\mathrm{x}$ & $\mathrm{x}$ & - \\
\hline $\mathrm{C}_{24} \mathrm{Cl}_{11}$ & 54 & 14.11 & $\mathrm{x}$ & - & $\mathrm{x}$ & - \\
\hline $\mathrm{C}_{24} \mathrm{Cl}_{12}$ & 57 & 14.29 & $\mathrm{x}$ & - & $\mathrm{x}$ & - \\
\hline $\mathrm{C}_{25} \mathrm{Cl}_{4}$ & 29 & 13.34 & $\mathrm{x}$ & - & $\mathrm{x}$ & $\mathrm{x}$ \\
\hline $\mathrm{C}_{25} \mathrm{Cl}_{5}$ & 34 & 13.52 & $\mathrm{x}$ & $\mathrm{x}$ & $\mathrm{x}$ & $\mathrm{x}$ \\
\hline $\mathrm{C}_{25} \mathrm{Cl}_{6}$ & 38 & 13.7 & $\mathrm{x}$ & $\mathrm{x}$ & $\mathrm{x}$ & $\mathrm{x}$ \\
\hline $\mathrm{C}_{25} \mathrm{Cl}_{7}$ & 42 & 13.88 & $\mathrm{x}$ & $\mathrm{x}$ & $\mathrm{x}$ & $\mathrm{x}$ \\
\hline $\mathrm{C}_{25} \mathrm{Cl}_{8}$ & 45 & 14.06 & $\mathrm{x}$ & $\mathrm{x}$ & $\mathrm{x}$ & $\mathrm{x}$ \\
\hline $\mathrm{C}_{25} \mathrm{Cl}_{9}$ & 48 & 14.24 & $\mathrm{x}$ & $\mathrm{x}$ & $\mathrm{x}$ & $\mathrm{x}$ \\
\hline $\mathrm{C}_{25} \mathrm{Cl}_{10}$ & 51 & 14.42 & $\mathrm{x}$ & $\mathrm{x}$ & $\mathrm{x}$ & $\mathrm{x}$ \\
\hline
\end{tabular}




\begin{tabular}{|c|c|c|c|c|c|c|}
\hline $\mathrm{C}_{25} \mathrm{Cl}_{11}$ & 53 & 14.6 & $\mathrm{x}$ & $\mathrm{x}$ & $\mathrm{x}$ & - \\
\hline $\mathrm{C}_{25} \mathrm{Cl}_{12}$ & 56 & 14.78 & $\mathrm{x}$ & - & $\mathrm{x}$ & - \\
\hline $\mathrm{C}_{26} \mathrm{Cl}_{2}$ & 16 & 13.47 & $\mathrm{x}$ & - & $\mathrm{x}$ & - \\
\hline $\mathrm{C}_{26} \mathrm{Cl}_{3}$ & 23 & 13.65 & $\mathrm{x}$ & - & $\mathrm{x}$ & $\mathrm{x}$ \\
\hline $\mathrm{C}_{26} \mathrm{Cl}_{4}$ & 28 & 13.83 & $\mathrm{x}$ & $\mathrm{x}$ & $\mathrm{x}$ & $\mathrm{x}$ \\
\hline $\mathrm{C}_{26} \mathrm{Cl}_{5}$ & 33 & 14.01 & $\mathrm{x}$ & $\mathrm{x}$ & $\mathrm{x}$ & $\mathrm{x}$ \\
\hline $\mathrm{C}_{26} \mathrm{Cl}_{6}$ & 37 & 14.19 & $\mathrm{x}$ & $\mathrm{x}$ & $\mathrm{x}$ & $\mathrm{x}$ \\
\hline $\mathrm{C}_{26} \mathrm{Cl}_{7}$ & 41 & 14.37 & $\mathrm{x}$ & $\mathrm{x}$ & $\mathrm{x}$ & $\mathrm{x}$ \\
\hline $\mathrm{C}_{26} \mathrm{Cl}_{8}$ & 44 & 14.55 & $\mathrm{x}$ & $\mathrm{x}$ & $\mathrm{x}$ & $\mathrm{x}$ \\
\hline $\mathrm{C}_{26} \mathrm{Cl}_{9}$ & 47 & 14.73 & $\mathrm{x}$ & $\mathrm{x}$ & $\mathrm{x}$ & $\mathrm{x}$ \\
\hline $\mathrm{C}_{26} \mathrm{Cl}_{10}$ & 50 & 14.91 & $\mathrm{x}$ & $\mathrm{x}$ & $\mathrm{x}$ & - \\
\hline $\mathrm{C}_{26} \mathrm{Cl}_{11}$ & 52 & 15.1 & $\mathrm{x}$ & $\mathrm{x}$ & $\mathrm{x}$ & - \\
\hline $\mathrm{C}_{26} \mathrm{Cl}_{12}$ & 55 & 15.28 & $\mathrm{x}$ & - & $\mathrm{x}$ & - \\
\hline $\mathrm{C}_{27} \mathrm{Cl}_{2}$ & 16 & 13.96 & $\mathrm{x}$ & - & $\mathrm{x}$ & $\mathrm{x}$ \\
\hline $\mathrm{C}_{27} \mathrm{Cl}_{3}$ & 22 & 14.14 & $\mathrm{x}$ & - & $\mathrm{x}$ & $\mathrm{x}$ \\
\hline $\mathrm{C}_{27} \mathrm{Cl}_{4}$ & 27 & 14.32 & $\mathrm{x}$ & - & $\mathrm{x}$ & $\mathrm{x}$ \\
\hline $\mathrm{C}_{27} \mathrm{Cl}_{5}$ & 32 & 14.50 & $\mathrm{x}$ & $\mathrm{x}$ & $\mathrm{x}$ & $\mathrm{x}$ \\
\hline $\mathrm{C}_{27} \mathrm{Cl}_{6}$ & 36 & 14.68 & $\mathrm{x}$ & $\mathrm{x}$ & $\mathrm{x}$ & $\mathrm{x}$ \\
\hline $\mathrm{C}_{27} \mathrm{Cl}_{7}$ & 40 & 14.86 & $\mathrm{x}$ & $\mathrm{x}$ & $\mathrm{x}$ & $\mathrm{x}$ \\
\hline $\mathrm{C}_{27} \mathrm{Cl}_{8}$ & 43 & 15.05 & $\mathrm{x}$ & $\mathrm{x}$ & $\mathrm{x}$ & $\mathrm{x}$ \\
\hline $\mathrm{C}_{27} \mathrm{Cl}_{9}$ & 46 & 15.23 & $\mathrm{x}$ & $\mathrm{x}$ & $\mathrm{x}$ & $\mathrm{x}$ \\
\hline $\mathrm{C}_{27} \mathrm{Cl}_{10}$ & 49 & 15.41 & $\mathrm{x}$ & $\mathrm{x}$ & $\mathrm{x}$ & - \\
\hline $\mathrm{C}_{27} \mathrm{Cl}_{11}$ & 51 & 15.59 & $\mathrm{x}$ & $\mathrm{x}$ & $\mathrm{x}$ & - \\
\hline $\mathrm{C}_{27} \mathrm{Cl}_{12}$ & 54 & 15.77 & $\mathrm{x}$ & - & $\mathrm{x}$ & - \\
\hline $\mathrm{C}_{28} \mathrm{Cl}_{2}$ & 15 & 14.45 & $\mathrm{x}$ & - & $\mathrm{x}$ & - \\
\hline $\mathrm{C}_{28} \mathrm{Cl}_{3}$ & 21 & 14.63 & $\mathrm{x}$ & - & $\mathrm{x}$ & - \\
\hline $\mathrm{C}_{28} \mathrm{Cl}_{4}$ & 27 & 14.81 & $\mathrm{x}$ & $\mathrm{x}$ & $\mathrm{x}$ & $\mathrm{x}$ \\
\hline $\mathrm{C}_{28} \mathrm{Cl}_{5}$ & 31 & 14.99 & $\mathrm{x}$ & $\mathrm{x}$ & $\mathrm{x}$ & $\mathrm{x}$ \\
\hline $\mathrm{C}_{28} \mathrm{Cl}_{6}$ & 35 & 15.18 & $\mathrm{x}$ & $\mathrm{x}$ & $\mathrm{x}$ & $\mathrm{x}$ \\
\hline $\mathrm{C}_{28} \mathrm{Cl}_{7}$ & 39 & 15.36 & $\mathrm{x}$ & $\mathrm{x}$ & $\mathrm{x}$ & $\mathrm{x}$ \\
\hline $\mathrm{C}_{28} \mathrm{Cl}_{8}$ & 42 & 15.54 & $\mathrm{x}$ & $\mathrm{x}$ & $\mathrm{x}$ & $\mathrm{x}$ \\
\hline $\mathrm{C}_{28} \mathrm{Cl}_{9}$ & 45 & 15.72 & $\mathrm{x}$ & $\mathrm{x}$ & $\mathrm{x}$ & $\mathrm{x}$ \\
\hline $\mathrm{C}_{28} \mathrm{Cl}_{10}$ & 48 & 15.90 & $\mathrm{x}$ & $\mathrm{x}$ & $\mathrm{x}$ & - \\
\hline $\mathrm{C}_{28} \mathrm{Cl}_{11}$ & 50 & 16.08 & $\mathrm{x}$ & $\mathrm{x}$ & $\mathrm{x}$ & - \\
\hline $\mathrm{C}_{28} \mathrm{Cl}_{12}$ & 53 & 16.26 & $\mathrm{x}$ & - & $\mathrm{x}$ & - \\
\hline $\mathrm{C}_{29} \mathrm{Cl}_{2}$ & 15 & 14.94 & $\mathrm{x}$ & - & $\mathrm{x}$ & - \\
\hline $\mathrm{C}_{29} \mathrm{Cl}_{3}$ & 21 & 15.12 & $\mathrm{x}$ & - & $\mathrm{x}$ & $\mathrm{x}$ \\
\hline $\mathrm{C}_{29} \mathrm{Cl}_{4}$ & 26 & 15.31 & $\mathrm{x}$ & - & $\mathrm{x}$ & $\mathrm{x}$ \\
\hline $\mathrm{C}_{29} \mathrm{Cl}_{5}$ & 31 & 15.49 & $\mathrm{x}$ & $\mathrm{x}$ & $\mathrm{x}$ & $\mathrm{x}$ \\
\hline $\mathrm{C}_{29} \mathrm{Cl}_{6}$ & 35 & 15.67 & $\mathrm{x}$ & $\mathrm{x}$ & $\mathrm{x}$ & $\mathrm{x}$ \\
\hline $\mathrm{C}_{29} \mathrm{Cl}_{7}$ & 38 & 15.85 & $\mathrm{x}$ & $\mathrm{x}$ & $\mathrm{x}$ & $\mathrm{x}$ \\
\hline $\mathrm{C}_{29} \mathrm{Cl}_{8}$ & 41 & 16.03 & $\mathrm{x}$ & $\mathrm{x}$ & $\mathrm{x}$ & $\mathrm{x}$ \\
\hline $\mathrm{C}_{29} \mathrm{Cl}_{9}$ & 44 & 16.21 & $\mathrm{x}$ & $\mathrm{x}$ & $\mathrm{x}$ & $\mathrm{x}$ \\
\hline $\mathrm{C}_{29} \mathrm{Cl}_{10}$ & 47 & 16.39 & $\mathrm{x}$ & $\mathrm{x}$ & $\mathrm{x}$ & - \\
\hline
\end{tabular}




\begin{tabular}{lllllll}
\hline \hline $\mathrm{C}_{29} \mathrm{Cl}_{11}$ & 50 & 16.57 & $\mathrm{x}$ & - & $\mathrm{x}$ & - \\
\hline $\mathrm{C}_{29} \mathrm{Cl}_{12}$ & 52 & 16.75 & $\mathrm{x}$ & - & $\mathrm{x}$ & - \\
\hline $\mathrm{C}_{30} \mathrm{Cl}_{2}$ & 14 & 15.44 & $\mathrm{x}$ & - & $\mathrm{x}$ & - \\
\hline $\mathrm{C}_{30} \mathrm{Cl}_{3}$ & 20 & 15.62 & $\mathrm{x}$ & - & $\mathrm{x}$ & - \\
\hline $\mathrm{C}_{30} \mathrm{Cl}_{4}$ & 25 & 15.80 & $\mathrm{x}$ & - & $\mathrm{x}$ & $\mathrm{x}$ \\
\hline $\mathrm{C}_{30} \mathrm{Cl}_{5}$ & 30 & 15.98 & $\mathrm{x}$ & $\mathrm{x}$ & $\mathrm{x}$ & $\mathrm{x}$ \\
\hline $\mathrm{C}_{30} \mathrm{Cl}_{6}$ & 34 & 16.16 & $\mathrm{x}$ & $\mathrm{x}$ & $\mathrm{x}$ & $\mathrm{x}$ \\
\hline $\mathrm{C}_{30} \mathrm{Cl}_{7}$ & 37 & 16.34 & $\mathrm{x}$ & $\mathrm{x}$ & $\mathrm{x}$ & $\mathrm{x}$ \\
\hline $\mathrm{C}_{30} \mathrm{Cl}_{8}$ & 41 & 16.52 & $\mathrm{x}$ & $\mathrm{x}$ & $\mathrm{x}$ & $\mathrm{x}$ \\
\hline $\mathrm{C}_{30} \mathrm{Cl}_{9}$ & 44 & 16.70 & $\mathrm{x}$ & $\mathrm{x}$ & $\mathrm{x}$ & $\mathrm{x}$ \\
\hline $\mathrm{C}_{30} \mathrm{Cl}_{10}$ & 46 & 16.88 & $\mathrm{x}$ & $\mathrm{x}$ & $\mathrm{x}$ & - \\
\hline $\mathrm{C}_{30} \mathrm{Cl}_{11}$ & 49 & 17.06 & $\mathrm{x}$ & $\mathrm{x}$ & $\mathrm{x}$ & - \\
\hline $\mathrm{C}_{30} \mathrm{Cl}_{12}$ & 51 & 17.24 & - & - & $\mathrm{x}$ & - \\
\hline $\mathrm{C}_{31} \mathrm{Cl}_{2}$ & 14 & 15.93 & $\mathrm{x}$ & - & $\mathrm{x}$ & $\mathrm{x}$ \\
\hline $\mathrm{C}_{31} \mathrm{Cl}_{3}$ & 20 & 16.11 & $\mathrm{x}$ & - & $\mathrm{x}$ & $\mathrm{x}$ \\
\hline $\mathrm{C}_{31} \mathrm{Cl}_{4}$ & 25 & 16.29 & $\mathrm{x}$ & - & $\mathrm{x}$ & $\mathrm{x}$ \\
\hline $\mathrm{C}_{31} \mathrm{Cl}_{5}$ & 29 & 16.47 & $\mathrm{x}$ & - & $\mathrm{x}$ & $\mathrm{x}$ \\
\hline $\mathrm{C}_{31} \mathrm{Cl}_{6}$ & 33 & 16.65 & $\mathrm{x}$ & $\mathrm{x}$ & $\mathrm{x}$ & $\mathrm{x}$ \\
\hline $\mathrm{C}_{31} \mathrm{Cl}_{7}$ & 37 & 16.83 & $\mathrm{x}$ & $\mathrm{x}$ & $\mathrm{x}$ & $\mathrm{x}$ \\
\hline $\mathrm{C}_{31} \mathrm{Cl}_{8}$ & 40 & 17.01 & $\mathrm{x}$ & $\mathrm{x}$ & $\mathrm{x}$ & $\mathrm{x}$ \\
\hline $\mathrm{C}_{31} \mathrm{Cl}_{9}$ & 43 & 17.19 & $\mathrm{x}$ & $\mathrm{x}$ & $\mathrm{x}$ & $\mathrm{x}$ \\
\hline $\mathrm{C}_{31} \mathrm{Cl}_{10}$ & 45 & 17.37 & $\mathrm{x}$ & $\mathrm{x}$ & $\mathrm{x}$ & - \\
\hline $\mathrm{C}_{31} \mathrm{Cl}_{11}$ & 48 & 17.55 & $\mathrm{x}$ & $\mathrm{x}$ & $\mathrm{x}$ & - \\
\hline $\mathrm{C}_{31} \mathrm{Cl}_{12}$ & 50 & 17.73 & $\mathrm{x}$ & - & $\mathrm{x}$ & - \\
\hline & & & & & \\
\hline
\end{tabular}

